\title{
Consequence of Adsorption Chromatography
}

\author{
Taleuzzaman $\mathbf{M}^{1} *$ and Soni $\mathrm{A}^{2}$ \\ ${ }^{1}$ Glocal School of Pharmacy, Glocal University, India \\ ${ }^{2}$ G.V.M. College of Pharmacy, India
}

*Corresponding author: Mohamad Taleuzzaman, Glocal School of Pharmacy, Glocal

University, Saharanpur, Mirzapur Pole 247121 UP, India, Email:

Editorial

Volume 2 Issue 3

Received Date: November 24, 2018

Published Date: December 10, 2018

DOI: $10.23880 /$ oajpr-16000165 zzaman007@gmail.com

\section{Editorial}

Chromatography classify based on separation mechanism, Adsorption chromatography is one type that is developed first, where the stationary phase has solid and mobile phase has a liquid or gaseous. When drugs are manufactured in pharmaceutical industry, the main object needs to sure before the product come into the market is that the product only contains the molecules that there are meant to be or it may be serious for the health. In Analytical field, purification is mainly done by chromatography. The HPLC (High-Performance Liquid Chromatography) especially is the main instruments based on adsorption chromatography to analyze what molecules a mixture consists of. A mixture can be divided in-fractions, which can then be analyzed with e.g. the MALDI-TOF analysis. The molecules separate due to some of the molecules in the mobile phase interact with the surface in the adsorption chromatography, they are absorbed by the surface. The first quantitative observations were carried out by C. W. Scheele in 1773 and F. Fontana in 1777.

The Figure 1 describes how a chromatography system works. The molecule in the mixture gathers with the stationary phase; therefore, slows them down. Because some particles move slower than other, that lead to separation. Chromatography system has a dynamic equilibrium as the separation between the two phases that is solid and liquid. The molecules' attraction towards the stationary phase and detachment from the stationary phase leading to the separation of a different molecule. In adsorption processes, the pores on the surface largely depend on their sizes. The current adsorption theory and relevant applications initiated by Langmuir's fundamental work have been developed extensively during the last 80 years. Presently, they comprise very advanced approaches that include a wide spectrum of modern surface chemistry.

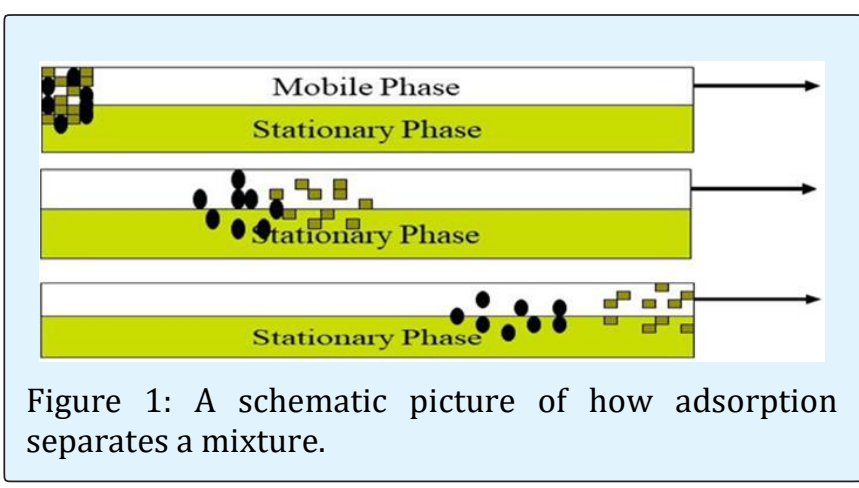

The current status of adsorption has been explained by a wide range of theoretical descriptions as well as by several practical examples, including industrial and environmental tasks. The crucial role of molecular modeling to understand adsorption phenomena and to develop novel classes of adsorbent materials has proven to be extremely valuable if used in conjunction with experimental techniques. This pattern will definitely continue and computer modeling will increasingly yield results same with those from experiments. For a practical point of view, it creates a stressed that produces new theoretical approaches and new groups of adsorbents generate new practical applications. Such a new concept produces a whole set of challenges and issues towards adsorption and related domain including catalysis, membrane separation, ion -exchange and process scale chromatography. Start the new branches that deal with 


\section{Open Access Journal of Pharmaceutical Research}

the suitable technologies that lessen the ecological load, introduce viable energy sources and allow for a plan for selection and search for ecology-friendly processes as well as the formulation of benchmark for estimation of the goodness of current chemical technologies and for design and production of new revolutionary solid materials.

It is globally known, that widely-understood adsorption science has acquired a dominating role in the modern pharmaceutical industry under environmental, economic and energy saving aspects. Certainly, adsorption technologies are fastly improved and adapted to extant tasks of mankind. With the help of scientist and engineers from both field industry and environment to develop the theory of adsorption science and to produce new adsorbents, catalysts and other advanced solids of great practical importance.

The ideal adsorbent must fulfill with the following criteria.

a. Adsorbent not soluble in the mobile phase.

b. For colored mixtures, adsorbents are must be colorless.

c. Particle size must be suitable to get a good separation and optimum flow rate.

d. Must be inert to solutes.

\section{Silica Gel}

It is commonly used as an adsorbent in column and thin layer Chromatography as well. The process used for the preparation of silica gel is by first by acidification of sodium silicate with sulphuric acid followed by washing with water and drying. A "Silanol groups" know which is the active sites of silica gel, hydroxyl groups attached to silicon atoms. The activity of the adsorbent reaches its maximum power when is heated between $150-250^{\circ} \mathrm{C}$ for removing the water. Removing water from adsorbent is necessary because if it presents, the mechanism of separation is a partition, not adsorption. The role particle size is very important, decreasing the particle size, increasing the surface area and, thus, increasing the separation power. Another important point with silica gel if the $-\mathrm{OH}$ group attached with an aliphatic chain of the silica gel is called silylation and commercial it is known as reversed Phase.

\section{Alumina}

Aluminum oxide $\left(\mathrm{Al}_{2} \mathrm{O}_{3}\right)$, is a common name, activated by heating at $400^{\circ} \mathrm{C}$ overnight. As an adsorbent it has a wide variety of positive properties like large capacity, is insoluble, relatively inert and easy availability. Due to its strong positive field $\left(\mathrm{Al}^{+3}\right)$, the mechanism is different from silica, the influence of basic sites which affect easily the polarized compounds. First choice adsorbents of aromatics from olefins. With this adsorbent the disadvantages that are, may cause rearrangement and ring expansion of unsaturated compounds, it is not for base labile compounds, and it could react chemically with acidic compounds. Commercial alumina available is (Neutral alumina: $\mathrm{pH}=7.5$, Acidic alumina: $\mathrm{pH}=4$. It is prepared by washing aluminum oxide with $\mathrm{HCl}$ then with distilled water, Basic alumina: $\mathrm{pH}=10$. It is prepared by washing $\mathrm{NaOH}$ then distilled water).

After of study the most important adsorbents, now elaborate the importance of the adsorption chromatography techniques in the industry. Its relevance becomes greater and greater in scientific advances due to the purification and separation of intermediates in multistage syntheses. In the analytical field the development of efficient columns, that capable of specific chiral compounds from mixture. The stationary phase that is using for the separation of enantiomers is called chiral stationary phase and chromatography is chiral chromatography, In a mixture the different enantiomers can be separated on the basis of the reactivity with the chiral stationary phase thus, the separation can be carried out. It is exactly a variant of a column chromatography where the two enantiomers of the same analyte compound differ in affinity to the single-enantiomer stationary phase, and they exit the column at different times. 